\title{
Clarify and communicate policies
}

W hile the Centers for Disease Control and Prevention (CDC) guidelines do allow for the extended storage of sterilized surgical packs, the guidelines also describe specific requirements with respect to the preparation, sterilization and storage of surgical packs. These requirements emphasize the importance of proper storage conditions when attempting to maintain the sterility of surgical packs over extended periods of time. "Event-related factors that contribute to the contamination of a product include bioburden (i.e., the amount of contamination in the environment), air movement, traffic, location, humidity, insects, vermin, flooding, storage area space, open/closed shelving, temperature, and the properties of the wrap material “( $\mathrm{p} .75)^{1}$. The Guide states: "General principles of aseptic technique should be followed for all survival surgical procedures", including "sterilization of instruments, supplies, and implanted materials" (p118) . As it is probably not reasonable to expect that all preparation, sterilization and storage requirements will be adhered to in research laboratory spaces, a re-sterilization frequency of once every six months would be considered a reasonable request to help ensure that the sterility of surgical instruments is maintained in a laboratory setting.

If the re-sterilization policy provided to Dr. Reinhold originated within the Department of Animal Resources, and as a direct result was not broadly accepted as an IACUC policy, then it is reasonable that investigative staff may conclude that the policy does not apply to their laboratories. The Department of Animal Resources' policy on surgical pack re-sterilization should be re-reviewed by the IACUC, and if the Committee approves, the policy should be renamed in a way that underscores that it is an institutional policy and is intended to cover all animal research at Great Eastern. To further evaluate whether Dr. Reinhold's practices pose a threat to animal welfare the IACUC could request Dr. Reinhold's lab provide supporting documentation that the lab's surgical packs have been prepared, sterilized, and stored in a manner that has not resulted in contamination during storage. While Dr. Reinhold may still resist adhering to the institutional policy based on his knowledge of existing governmental guidelines, a collegial discussion with the IACUC and the veterinary staff can help to address the investigators concerns, and inviting Dr. Reinhold or Department of
Surgery staff to help develop or refine the institutional policy in cooperation with the veterinary and IACUC staff could help with future compliance.

Although veterinarians are the subject matter experts with respect to the use of aseptic technique in a laboratory animal setting, the IACUC should make an effort to work closely with the veterinarians, researchers and other stakeholders when developing survival surgical guidelines and policies, and to ensure that the scope of any policy generated is clearly communicated.

\section{Maria Reaves ${ }^{\star}$, Kevin Prestia and Skye Rasmussen NYU Langone Health, New York, NY, USA. \\ *e-mail:Maria.Reaves@nyulangone.org}

Published online: 23 October 2018 https://doi.org/10.1038/s41684-018-0178-5

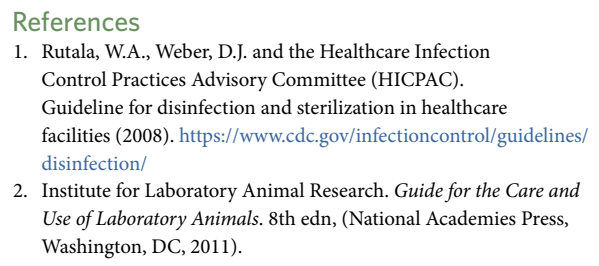

2. Institute for Laboratory Animal Research. Guide for the Care and Use of Laboratory Animals. 8th edn, (National Academies Press, Washington, DC, 2011).

\section{Review, revise, and clarify}

T he use of aseptic technique, including sterilized instrumentation, is a requirement for all survival surgeries ${ }^{1}$. If a surgical pack becomes contaminated, it clearly needs to be resterilized; however, there is no regulation governing the frequency required for re-sterilization of packs that are unopened and uncontaminated. In the case of Great Eastern University, the Department of Animal Resources had developed an internal policy requiring re-sterilization every six months, and this was approved by IACUC. It is not clear, though, whether this policy applied only to the Department of Animal Resources, or was meant to be a universitywide policy to which all researchers must adhere. If the latter, then the Reinhold lab may be held to that standard as an IACUC may set requirements that fall outside of, or are more stringent than, applicable guidelines and regulations.

Dr. Reinhold does make a case for his lab's policy of keeping packs indefinitely if they are unopened and uncontaminated. He notes the lack of surgical infections in his lab's surgical animals, and refers to a CDC report "Guideline for disinfection and sterilization in healthcare facilities (2008)"'2 to support his stance. One study within these Guidelines states: "Another [study] evaluated the effectiveness of eventrelated outdating by microbiologically testing sterilized items. During the 2-year study period, all of the items tested were sterile. Thus, contamination of a sterile item is event-related and the probability of contamination increases with increased handling." This document then goes on to include recommendations on proper handling and storage of medical and surgical devices following sterilization in order to prevent contamination. Some considerations noted in the guidelines include storage far enough from the floor, ceiling and outside walls to allow for air circulation, easing of cleaning and compliance with fire codes; and storing in locations where the packs will not become wet and contaminated. In this scenario from Great Eastern University, the method of storage was not delineated, but should also be taken into consideration when considering the sterility of the packs.

The IACUC has the authority to make the ultimate decision on whether this is a significant finding. Ideally that decision should be based on regulatory rules and guidelines, or an institution-wide IACUC policy based on literature or evidence supporting their policy. The use of performance standards are stressed in the "Guide for the Care and Use of Laboratory Animals" provide evidence that his surgical packs remain sterile based on the method of sterilization and proper storage conditions, the IACUC may consider changing its decision of a "significant deficiency", and revise their sterilization policy accordingly. If the policy is revised, it should be made clear that it applies to the whole institution, 